\title{
UNA BREVE LECTURA EN CLAVE INDÍGENA DE LA JURISPRUDENCIA CONSTITUCIONAL COLOMBIANA DEL 2019 SOBRE TRATADOS DE INVERSIÓN
}

A brief reading from the Colombian constitutional jurisprudence of 2019 about investment treaties from an indigenous peoples' rights perspective

PAULA ROBLEDO SILVA Universidad Externado de Colombia paula.robledo@uexternado.edu.co

DANIEL RIVAS-RAMÍREZ

Instituto Internacional de Derechos Humanos-Capítulo Colombia danielrivasram@hotmail.com

\section{SUMARIO}

I. INTRODUCCIÓN. II. INDÍGENAS, APERTURA E INVERSIÓN. III. ANTECEDENTES JURISPRUDENCIALES EN LA MATERIA. IV. EL PANORAMA JURISPRUDENCIAL DEL 2019: 1. La constitucionalidad condicionada del APPRI entre Colombia y Francia (Sentencia C-252 del 2019.) 2. La constitucionalidad del TLC entre Colombia e Israel (Sentencia C-254 del 2019) V. CONCLUSIONES. BibLIOGRAFíA. JuRISPRUDENCIA DE la Corte Constitucional colombiana.

\section{INTRODUCCIÓN}

Pese a que Colombia no puede ser catalogado como un país cuyo sistema jurídico sea dualista en lo que respecta a la relación con el derecho internacional, el ordenamiento constitucional prevé la figura de las leyes aprobatorias 
de tratados internacionales, a través de las cuales el Congreso aprueba o imprueba la ratificación de dichos acuerdos. En estricto sentido, el contenido de estas leyes es limitado, toda vez que se restringe a la aprobación del texto del tratado internacional y, por tanto, en realidad la sustancia permanece dentro del instrumento internacional y no dentro de la norma jurídica nacional (Acosta Alvarado, 2018: 173).

No obstante, el constituyente de 1991 previó que al tratarse de una ley aprobatoria, que pese a no tener contenido sustancial sigue siendo una ley, debía estar sometida a un examen de constitucionalidad. Es así como, dentro de las principales funciones que se atribuyeron a la Corte Constitucional colombiana, se incluyó la de llevar a cabo el control automático de constitucionalidad de este tipo de leyes; ideándola como un mecanismo encaminado a garantizar, por un lado, la salvaguarda de la supremacía constitucional y, por el otro, la coherencia (tanto interna como externa) del sistema normativo previsto en la Constitución.

Ahora bien, es importante aclarar que, pese a que se trata de un requisito procedimental, el control automático de constitucionalidad de este tipo de leyes se erige, dentro del ordenamiento jurídico nacional, como la última oportunidad para garantizar que los tratados internacionales celebrados por Colombia cumplan con los valores y principios previstos en la Constitución nacional. Esto en particular si se tiene en cuenta que dicha revisión se realiza antes de la sanción presidencial de la ley aprobatoria, pero, en todo caso, después de la negociación y suscripción del tratado por parte del Ejecutivo y de la deliberación para su aprobación por el Congreso de la República. En suma, se trata del último momento en el que, desde el engranaje institucional del Estado, es posible frenar, en cierto sentido, un tratado internacional contrario a los valores y los principios constitucionales.

Por lo anterior, las sentencias que versan sobre las leyes aprobatorias de tratados internacionales son un componente importante de la jurisprudencia constitucional que profiere la Corte Constitucional año tras año. Relevancia que es mayor en el caso de tratados internacionales particularmente sensibles como el de los Tratados de Libre Comercio y los Acuerdos de Protección y Promoción a la Inversión Extranjera (APPRIs), que generan importantes impactos socio-jurídicos al interior de los Estados receptores. Por esta razón, hemos elegido para la crónica jurisprudencial colombiana de este año el caso de las sentencias de constitucionalidad de las leyes aprobatorias de APPRIs con las cuales consideramos que la Corte Constitucional omitió valorar debidamente uno de los sectores más afectados por este tipo de tratados internacionales: los pueblos indígenas.

\section{INDÍGENAS, APERTURA E INVERSIÓN}


Dentro de los rasgos definitorios de la Constitución de 1991 podemos resaltar al menos tres que son importantes para entender la compleja tensión constitucional que surge entre la recepción de APPRIs y los derechos de los pueblos indígenas en Colombia. El primero de ellos es el reconocimiento del pluralismo y la participación ciudadana como principios fundamentales del Estado a partir de los cuales se derivan otras disposiciones relacionadas con la diversidad étnica y cultural que promueven la igualdad y participación de las minorías étnicas, como los pueblos indígenas. De allí que tanto a nivel constitucional y legal como jurisprudencial, figuras como la consulta previa, los derechos colectivos de los pueblos indígenas, el reconocimiento institucional y territorial de la autonomía indígena y los mecanismos de participación ciudadana, entre otros, tengan tanta relevancia en el contexto colombiano.

En paralelo a lo anterior, la Constitución también contempla una serie de elementos orientados a la consolidación de un modelo económico determinado que tradicionalmente ha sido asociado con el neoliberalismo: la economía social de mercado. Es por ello que la Constitución incluye un título dedicado exclusivamente al establecimiento de un régimen económico dentro del cual se protegen la libertad de competencia y la libertad de empresa, la función social de la propiedad privada, la intervención del Estado en la economía y, por supuesto, se promueve también la internacionalización de las relaciones económicas del Estado.

Corolario de lo anterior es que desde la expedición de la Carta de 1991 el Estado haya puesto en marcha una política económica tendente a internacionalizar la economía nacional a través de esquemas de liberalización del comercio y la atracción del capital extranjero. Es así, con fundamento en dicho objetivo, que Colombia ha tenido un crecimiento importante en cuanto a la celebración de TLCs y APPRIs hasta el punto de contar a día de hoy con más de treinta tratados internacionales de contenido económico.

Ahora bien, en principio, estos dos rasgos distintivos de la Constitución no parecerían ser prima facie contrarios entre sí, sin embargo, debido a factores como a la indebida negociación de este tipo de tratados internacionales (Tole Martínez, 2018: 208), la asimetría de poder y los sesgos colonialistas del sistema internacional económico (Prieto-Ríos y Rivas-Ramírez, 2020) y los efectos materiales que se producen en la ejecución de estos tratados, surge una innegable tensión entre los intereses económicos y los derechos de los pueblos indígenas. Tan solo a modo de ejemplo basta con señalar que en virtud de los APPRIs y los TLCs que Colombia ha firmado hasta el momento, numerosas empresas multinacionales han llegado al país para explotar recursos naturales que se encuentran en territorios indígenas y sus zonas de influencia sin importar la afectación que ello pueda suponer para los pueblos indígenas. Tanto es así que en casos como el de las multinacionales Cosigo y Tobie Mining v. Colombia no solo se aprecia que el proyecto de exploración y explotación minera afecta directamente los territorios de algunos pueblos indígenas de la Amazonía colombiana, sino que, además, se ha demostrado que la multinacional ha injerido en asuntos indígenas 
y ha fabricado su consentimiento para la realización del proyecto (Corte Constitucional, Sentencia T-384A del 2014).

Así las cosas, en virtud del mandato de la Corte Constitucional como guardián de la supremacía de la Constitución y máximo intérprete del texto constitucional, tiene la obligación de realizar una interpretación armónica del texto constitucional a través de la cual, en aquellos casos en donde pueda surgir una tensión de este tipo, la resuelva y fije pautas claras para evitar dichas contradicciones. En ese sentido, sería de esperar que en escenarios como el control de constitucionalidad de las leyes aprobatorias de los tratados internacionales de contenido económico como los TLCs y los APPRIs realizara tal ejercicio, teniendo presente los derechos de los pueblos indígenas como criterio o parámetro de constitucionalidad.

\section{ANTECEDENTES JURISPRUDENCIALES EN LA MATERIA}

En lo que respecta al caso de los APPRIs y TLCs con capítulos sobre inversión extranjera, la Corte Constitucional se ha pronunciado hasta el momento en veinticinco oportunidades (Rivas-Ramírez, 2020). A partir de una lectura transversal de esta jurisprudencia es posible identificar al menos tres conclusiones que resultan relevantes para los efectos de esta crónica y que versan sobre la forma en la que el juez constitucional ha llevado a cabo este tipo de control de constitucionalidad. En específico se trata de cuestiones relacionadas con: i) el papel que le ha otorgado al derecho internacional dentro del ordenamiento jurídico nacional, ii) el alcance que ha tenido dicho control de constitucionalidad y iii) sobre la inclusión y participación que dentro de estos procesos ha tenido la ciudadanía en general y los pueblos indígenas en particular.

El primer tema que nos interesa resaltar frente a la jurisprudencia constitucional sobre la exequibilidad de los tratados relativos a la inversión extranjera es la que tiene que ver con el papel que el juez constitucional colombiano les ha otorgado al interior del ordenamiento jurídico nacional. Tal y como señalábamos al principio, Colombia, lejos de ser un ordenamiento dualista, ha sido calificado como uno de corte monista moderado (Corte Constitucional, Sentencia C-400 de 1998), pese a que por lo general reivindica la supremacía de la Constitución nacional sobre el derecho internacional. En ese sentido, el juez constitucional suele entender que las normas internacionales deben guardar coherencia y respeto con la Constitución nacional y, por tanto, salvo que se integren al bloque de constitucionalidad, no pueden ser tenidas como parámetros de constitucionalidad.

En el caso de las normas internacionales en materia de inversión, la lógica es la misma. De acuerdo con la Corte Constitucional, los tratados internacionales de la materia que son aprobados por medio de una ley son 
revestidos, en principio, de una naturaleza legal (Sentencia C-155 del 2007). Esto quiere decir que dentro del andamiaje normativo están por debajo de la Constitución, pero al mismo nivel que las leyes; por lo que en oportunidades, pueden convertirse en mandatos de optimización (Sentencia C-309 del 2007). No obstante, en casos en los que hay una antinomia entre el tratado internacional y una de las directivas del Banco de la República, los primeros son revestidos de una naturaleza infrarreglamentaria $y$, por tanto, ni siquiera son tenidos como parámetro de legalidad (Rivas Ramírez, 2017: 654). En suma, la posición que la Corte ha sostenido con relación al papel que ocupan estas normas internacionales al interior de nuestro ordenamiento es que nunca podrá reconocérseles rango constitucional y, por tanto, ante una eventual colisión con disposiciones o principios constitucionales, estos últimos tendrían que prevalecer.

El segundo elemento que vale la pena resaltar con relación a la jurisprudencia constitucional en la materia es el que tiene que ver con la naturaleza y alcance del control de constitucionalidad que se hace a este tipo de tratados internacionales. Este control es de carácter preventivo, abstracto y definitivo (Corte Constitucional. Sentencia C-178 de 1995) y se limita a evaluar la congruencia de la ley aprobatoria y el tratado internacional con la Constitución política (Corte Constitucional. Sentencia C-750 del 2008). Más concretamente, se caracteriza por ser un control a priori de carácter débil por las siguientes razones. En primer lugar, parte del hecho de que los tratados analizados no constituyen un parámetro de constitucionalidad y por tanto no cobran efectos jurídicos plenos en el ordenamiento nacional. Se abstiene de cualquier consideración de carácter técnico u operativo o que esté relacionada con la conveniencia política o económica del acuerdo internacional. Y, finalmente, se limita a verificar que no haya un desdoblamiento de competencias constitucionales presidenciales y al cumplimiento de los principios de equidad, reciprocidad y conveniencia nacional que rigen el mandato constitucional de la internacionalización de las relaciones económicas del Estado (Rivas-Ramírez, 2018: 224-226). Esto quiere decir que, en realidad, se trata de un control de constitucionalidad principalmente formal que apenas utiliza de manera fragmentada la Constitución nacional.

Por último, un tercer rasgo que vale la pena resaltar de la jurisprudencia constitucional en la materia es el que tiene que ver con la inclusión y participación de la ciudadanía y de los pueblos indígenas en los procesos de constitucionalidad de las leyes aprobatorias de este tipo de tratados internacionales. En realidad, lo que interesa resaltar es que más allá del escenario de las intervenciones ciudadanas (que es obligatorio en virtud de la Constitución y del Reglamento de la Corte), en este tipo de procesos de constitucionalidad ha sido excepcional contar con la participación ciudadana, así como también ha sido extraño ver que el juez constitucional incluya a minorías étnicas a través de figuras como la consulta previa o por medio de una valoración subjetiva 
de sus condiciones de vulnerabilidad frente a los impactos que este tipo de tratados podrían suponer para ellas (Robledo Silva y Rivas-Ramírez, 2020).

En conclusión, el desarrollo jurisprudencial en la materia entre 1991 y 2018 nos permite concluir que no hay duda, de acuerdo con el criterio del juez constitucional, que los APPRIs tienen los mismos efectos jurídicos que una ley; que su control de constitucionalidad se limita a cuestiones formales de la negociación y suscripción del tratado y al cumplimiento de los principios rectores de las relaciones internacionales del Estado y que en dichos procesos rara vez se da relevancia a la ciudadanía, inclusive cuando esta se vea directamente afectada por la ratificación de este tipo de acuerdos internacionales.

\section{EL PANORAMA JURISPRUDENCIAL DEL 2019}

En el año 2019, la Corte Constitucional adoptó dos nuevas decisiones en el marco del control automático de constitucionalidad sobre leyes aprobatorias de tratados internacionales de contenido económico. La primera de ellas fue la sentencia C-252, por medio de la cual declaró la exequibilidad condicionada del Acuerdo para la Promoción y Protección Recíprocas de Inversiones entre Colombia y Francia, firmado en julio del 2014. La segunda fue la sentencia C-254 del 2019, con la que declaró la constitucionalidad del acuerdo de libre comercio celebrado entre Colombia e Israel el 13 de noviembre del 2015 y que contiene un capítulo específico sobre la inversión extranjera.

A grandes rasgos ambos acuerdos mantienen la estructura genérica de los tratados internacionales de su clase. En ese sentido, el acuerdo celebrado entre Colombia y Francia mantiene la estructura y contenido fundamentales de cualquier otro APPRI y el instrumento acordado entre Colombia e Israel, el de cualquier otro TLC. No obstante lo anterior, tanto en uno como en otro surgieron algunas cuestiones problemáticas para la opinión pública en el contexto nacional colombiano, las cuales situaron sus procesos de control de constitucionalidad en el foco de la atención pública.

En términos generales, esto ocurrió en la medida en la que, pese a que Colombia acuñó constitucionalmente un modelo económico neoliberal que tiende hacia la internacionalización y a la liberalización de la economía, la experiencia de los últimos años ha contribuido a la consolidación de una oposición generalizada de la ciudadanía a este tipo de procesos a raíz de los impactos sociales, económicos y tributarios que tienen para la población. Así, por ejemplo, a raíz del TLC con Estados Unidos gran parte de la población ha empezado a mostrar su desacuerdo con la internacionalización de las relaciones económicas del Estado como consecuencia de los impactos que dicho acuerdo tuvo para los productores nacionales. De igual manera, durante los últimos cinco años se ha despertado una preocupación generalizada en contra de los APPRIs en la medida en la que Colombia ha sido demandada 
internacionalmente en múltiples oportunidades a raíz de decisiones adoptadas por las autoridades públicas en pro del interés público nacional, dentro de las cuales estaba incluso la protección de los pueblos indígenas del sur del país. A continuación reseñaremos brevemente ambas decisiones a partir de la tensión constitucional que surge entre la adopción de este tipo de acuerdos y los derechos de los pueblos indígenas.

\section{LA CONSTITUCIONALIDAD CONDICIONADA DEL APPRI ENTRE COLOMBIA Y FRANCIA (SENTENCIA C-252 DEL 2019)}

Como hemos señalado, el APPRI celebrado entre Colombia y Francia tenía, grosso modo, la misma estructura y contenido de los demás tratados de este tipo que Colombia ha ratificado en el pasado. Sin embargo, dentro de su articulado incluía una serie de cláusulas cuya redacción abría la puerta para interpretaciones que podrían resultar en detrimento de los intereses nacionales de Colombia, como es el caso del art. 15 sobre la solución de diferencias relativas a la inversión o el art. 16 en donde se incluía una disposición en la que se adoptaba una especie de expansión de la cláusula de la nación más favorecida respecto a cualquier obligación derivada del derecho internacional. Sin embargo, el juez constitucional intentó hacer un análisis cuidadoso de este tipo de cláusulas y tomó una decisión al respecto.

El resultado de este proceso, como hemos anticipado, fue la declaratoria de constitucionalidad condicionada de la ley aprobatoria y del tratado; una decisión histórica en la medida en la que por primera vez en la materia, el juez constitucional moduló el alcance de este tipo de tratados internacionales y advirtió al presidente de la República a adelantar las gestiones necesarias para adoptar una declaración interpretativa del tratado internacional. Más concretamente, el juez constitucional estableció cómo debían ser interpretadas determinadas expresiones del tratado, tales como «de conformidad con el derecho internacional aplicable a los inversionistas de la otra parte contratante y a sus inversiones en el territorio», «expectativas legítimas» $\mathrm{y}$ "necesarias y proporcionales» para evitar la generación de interpretaciones contrarias a la Constitución nacional.

A diferencia de como había ocurrido en el pasado con la revisión automática de constitucionalidad, la Corte Constitucional decidió desplegar un impresionante esfuerzo de transparencia y participación ciudadana, abriendo diferentes escenarios que trascendían la simple participación a través de las intervenciones ciudadanas ${ }^{1}$. Fue así como no solo recibió y valoró detenidamente dichos escritos ciudadanos, sino que además realizó una

Hay que recordar que, por mandato de la Constitución y del Reglamento Interno de la Corte, en el marco de los procesos de constitucionalidad, siempre debe abrirse 
audiencia pública extensa en la que consultó y dialogó con expertos nacionales de diferentes orientaciones sobre el contenido y los posibles efectos de este tratado. Así mismo, cambió también la tradición en lo que respecta al análisis de constitucionalidad de estos tratados en la medida en la que, pese al objeto y alcance limitado que tiene este control de constitucionalidad, realizó un análisis detenido, profundo y detallado de cada una de las cláusulas que contenía el tratado; valorando también los argumentos esgrimidos tanto por los intervinientes ciudadanos como por los expertos invitados por la Corte. En realidad, en los anteriores procesos de este tipo, la Corte se limitaba a realizar un análisis en bloque y superfluo frente a los arts. 9, 226 y 227 de la Constitución, por lo que esta decisión ha llevado a que algunos doctrinantes aseguren que hay un cambio en cuanto al tipo de control que hace la Corte, hasta el punto de afirmar que se trata de un cambio de paradigma orientado a que el juez constitucional complementa de manera sutil el proceso de toma de decisiones del Poder Ejecutivo sin que con ello genere un desequilibrio de poderes (Tamayo Álvarez, 2020, 472).

Al respecto debemos señalar que en esta sentencia el juez constitucional hace un esfuerzo por hacer un análisis más complejo y completo de la cuestión, hasta el punto de utilizar herramientas que tradicionalmente no han sido propias de la labor del juez constitucional colombiano. Es así como encontramos que la Corte utiliza fuentes y disposiciones propias de derecho internacional como parámetro de análisis hasta el punto en que las utiliza y aplica de manera directa en su análisis e, incluso, intenta lidiar con fenómenos como la fragmentación del derecho internacional. Así, entonces, encontramos que en la sentencia C-252 del 2019 usa instrumentos como la Convención Americana sobre los Derechos Humanos (que no es una norma propia del derecho internacional de la inversión) para interpretar el alcance y los impactos que tiene el APPRI en el contexto colombiano. Así mismo, utiliza arbitrajes internacionales de inversión para interpretar y develar el sentido y alcance de las cláusulas de protección que contiene el tratado, como ocurre cuando trae a colación el caso Occidental c. Ecuador para analizar la cláusula de trato nacional, o el caso CMS Gas Transmissin Co c. Argentina para interpretar la cláusula de trato arbitrario o discriminatorio.

No obstante, con respecto a esto último, debemos señalar que pese a que se trata de un buen intento, el uso de este tipo de herramientas como los arbitrajes internacionales de inversión termina conduciendo a que el análisis del juez constitucional pierda rigor y precisión en la medida en la que los utiliza de manera indiscriminada, sin fundamento metodológico y desconociendo las vicisitudes técnicas y jurídicas que tienen este tipo de decisiones. Así, por ejemplo, el magistrado Linares señala acertadamente en su aclaración

espacio para la recepción de intervenciones ciudadanas que defiendan o contradigan la exequibilidad de la norma analizada. 
y salvamento parcial de voto que las decisiones de los tribunales de arbitraje internacional de inversión no pueden ser tenidas en cuenta como precedentes jurisprudenciales, tal y como lo hace la Corte en el análisis que realiza; y que tampoco pueden ser utilizadas de manera indistinta e indiscriminada solo porque desarrollen una interpretación respecto a una cláusula concreta. En realidad, estas deben ser utilizadas con mucha cautela en la medida en la que los supuestos y condiciones de cada arbitraje internacional de inversión son diferentes $y$, sin tal cuidado, podría terminarse utilizando como elemento de análisis una decisión que podría no ser análoga a la situación o el contexto colombiano.

Sumado a lo anterior, la sentencia, o más bien el proceso de constitucionalidad adelantado, tiene otra serie de cuestiones problemáticas sobre las cuales - por cuestiones de espacio - no nos pronunciaremos a fondo. Sin embargo, sí hay una que nos parece importante resaltar y comentar con algo más de detalle: la exclusión de los pueblos indígenas.

Como hemos señalado al principio de estas páginas, Colombia es un Estado social de derecho que dentro de sus valores y principios se encuentra el pluralismo constitucional y con ello, el reconocimiento y protección de los pueblos indígenas en las distintas esferas de la sociedad. Esto quiere decir, entre otras cosas, que los pueblos indígenas deben ser tenidos en cuenta en las decisiones que les afectan, así como también que están revestidos de una especial protección constitucional debido a su histórica discriminación y vulneración de derechos. En el contexto del trabajo de la Corte Constitucional esto quiere decir que ella, como máxima guardiana de la Constitución nacional, debe tener presente ambas cosas en todo momento y garantizar que los intereses y derechos de los pueblos indígenas sean valorados y protegidos según cada caso; inclusive, si se trata de una decisión sobre la constitucionalidad de un ley o un tratado internacional. Es decir, que, en principio, el juez constitucional debería haber considerado esta cuestión en el marco de su decisión sobre el APPRI entre Colombia y Francia, al menos en tres momentos.

En primer lugar, el juez constitucional debería haber propiciado su participación activa como intervinientes en el proceso de constitucionalidad. Después de ello, debería haber analizado la necesidad y legalidad de la consulta previa frente a la suscripción de este tratado internacional. Y por último, tendría que haber incorporado dentro del análisis de constitucionalidad una perspectiva no universalista de los derechos fundamentales y de la autodeterminación de los pueblos. Veamos un poco más en detalle cada una de estas cuestiones.

Con relación a la participación de los pueblos indígenas en el proceso de revisión de constitucionalidad de la ley aprobatoria debemos señalar que, en este caso en concreto, el juez constitucional omitió hacerlo. Si bien es cierto que los pueblos indígenas no participaron voluntariamente a través de un escrito de intervención ciudadana, esto no exime al juez constitucional 
de haberles invitado a participar en el proceso, tal y como bien hizo con otros sectores, organizaciones y expertos cuyos intereses se verían afectados - positiva o negativamente- por el tratado; en particular en un proceso que, como hemos dicho, fue tan abierto y tan participativo. Al respecto debemos anotar también que el juez constitucional no puede perder de vista que en el contexto colombiano siempre ha existido una marginalización de la voluntad indígena, especialmente en cuestiones relacionadas con temas económicos y cuya filigrana es técnica y compleja (Robledo Silva y Rivas-Ramírez, 2020).

Con relación a la consulta previa es menester aclarar que en este caso el juez constitucional sí se detuvo a considerar y analizar si era o no necesario adelantarla. Por su parte, a juicio de la Corte, la consulta no era necesaria en la medida en que no se trataba de una decisión administrativa o legislativa que afectara de manera «directa» los intereses de los pueblos indígenas. No obstante lo anterior, a nuestro criterio esto es discutible en la medida en la que la misma Corte Constitucional ha sostenido en su jurisprudencia que el interés «directo» debe ser entendido relativamente; por lo que, en un caso como este, sobre el cual además hay evidencia fáctica y jurídica que demuestra el potencial negativo que tienen los APPRI sobre los pueblos indígenas, tendría que haber sido reconocido su interés directo.

Sumado a lo anterior debemos señalar que, en realidad, el uso de la consulta previa en contextos de negociación y celebración de tratados internacionales de contenido económico no es extraño. Así, por ejemplo, países latinoamericanos como Perú y México y países de otras latitudes como Canadá y Nueva Zelanda han hecho de la consulta previa un requisito para la toma de decisiones de política exterior que tengan la capacidad de afectar a los pueblos indígenas; tal y como ocurrió, por ejemplo, en el caso de la vinculación de estos últimos dos países a la Alianza del Pacífico o la suscripción del UMSCA (Robledo Silva y Rivas-Ramírez, 2019; Prieto-Ríos y Rivas-Ramírez, 2020).

Finalmente, y con relación a los usos e interpretaciones universalistas del derecho, debemos señalar que en el caso de la sentencia C-252 del 2019 son frecuentes en la medida en la que, por el lenguaje y los contextos a través de los cuales justificó la ausencia de participación de los pueblos indígenas, así lo demuestra. Así, por ejemplo, el hecho de que se haya considerado superado el proceso de diálogo con los pueblos indígenas a partir de una simple socialización del proceso de celebración del tratado muestra que la Corte cae en dichos sesgos universalistas. $\mathrm{Al}$ respecto, basta con señalar que escenarios de socialización no son otra cosa que mecanismos que pretenden legitimar las decisiones a través de una falsa inclusión de la voluntad indígena sin que con ello se preste atención, se valore y se integre sustancialmente la opinión y la posición de los pueblos indígenas; lo que no es otra cosa más que un derivado del sesgo colonial de nuestros ordenamientos jurídicos y sociales que se manifiesta a través de la lógica de la asimilación (Merino, 2018: 465).

En suma, con relación a la sentencia C-252 del 2019 podemos concluir que, pese a que se trata de una decisión hito en la jurisprudencia constitucional 
colombiana en materia de tratados de inversión, contiene también una multiplicidad de elementos problemáticos e incluso discriminatorios que conducen a una falla en el control de constitucionalidad y, lo que es más grave, en la función especial que tiene la Corte Constitucional respecto a la protección y salvaguarda de los pilares fundamentales del Estado colombiano; situación que se evidencia de manera cristalina en el contexto de los pueblos indígenas.

\section{LA CONSTITUCIONALIDAD DEL TLC ENTRE COLOMBIA E ISRAEL (SENTENCIA C-254 DEL 2019)}

La segunda y última sentencia de la Corte Constitucional que fue adoptada en el año 2019 con relación a tratados de contenido económico que versaran sobre inversión extranjera es la sentencia C-254. En ella, el juez constitucional analizó y declaró la constitucionalidad del TLC celebrado entre Colombia e Israel. En ella, a diferencia de como ocurrió con el proceso de la C-252 del 2019, la Corte no asumió un papel innovador, sino que, por el contrario, se limitó a realizar el control de constitucionalidad con base en el paradigma tradicional. En ese sentido, la sentencia se limita, en buena medida, a realizar un control limitado de constitucionalidad en el que hace un contraste en bloque y superfluo del tratado; especialmente en lo que tiene que ver con la inversión extranjera.

En lo que respecta a la participación de los pueblos indígenas y la inclusión de sus intereses en esta decisión, debemos señalar que, en realidad, ocurre prácticamente lo mismo que con la C-252 del 2019. No hay participación de los pueblos indígenas en la fase pública del proceso, se desestima la consulta previa y se mantiene un discurso - probablemente inconsciente- universalista. Al respecto, y con el objetivo de hacer un análisis conjunto de estas tres cuestiones, es importante señalar que en la sentencia se utilizan tres conceptos claves que sintetizan de manera clara el problema del ejercicio adelantado por la Corte Constitucional en este caso. Se trata de las expresiones «sociedad en general», «socialización» y «autodeterminación de los pueblos».

Con respecto a ellas, hemos de anotar que el juez constitucional, en lugar de hacer un uso cuidadoso y reflexivo de cada una de ellas, las utiliza de manera indistinta para justificar su posición respecto a ignorar la posición especial que los pueblos indígenas podrían tener respecto a la celebración de este TLC. Más concretamente, utiliza la expresión «sociedad en general» para fundamentar su decisión de considerar necesaria la consulta previa en la medida en la que el tratado afecta a la sociedad en general y no de manera específica a los pueblos indígenas; ignorando con ello que los pueblos indígenas como sujetos de especial protección enfrentan barreras y afectaciones diferenciales que merecen una consideración particular. De igual manera, justifica la falta de consulta previa a través de la socialización del tratado en consultas públicas generales, en donde se repite el mismo problema que hemos detectado y 
explicado en el contexto de la C-252 del 2019. Finalmente, hace uso del concepto de «autodeterminación de los pueblos» para justificar la celebración del tratado internacional; lo cual, si bien es cierto que podría ser tenido como un uso correcto de la figura, lo hace desde una perspectiva universalista en la que ignora la plurinacionalidad que existe en Colombia y, por tanto, somete a los pueblos indígenas a la voluntad popular «occidental», blanca y hegemónica; eliminando cualquier espacio para su participación en ella.

Es así como a partir de la lectura y análisis de la sentencia C-254 del 2019 es posible intuir que, en realidad, cuando se trata de cuestiones que involucran intereses económicos y elementos de la política económica de apertura del Estado, existe una posición institucional de la Corte respecto a la vinculación y participación de los pueblos indígenas. Sin embargo, también revela, de manera preocupante, que inclusive en este Alto Tribunal podría existir una consciencia inconsciente sobre el universalismo del derecho que reproduce patrones y lógicas coloniales y excluyentes.

\section{CONCLUSIONES}

Como hemos visto al principio de este texto, Colombia es un Estado social de derecho cuya configuración constitucional implica un sistema complejo de valores y principios dentro de los que se encuentran, por un lado, el pluralismo y la diversidad cultural y, por el otro, un modelo económico abierto y neoliberal. Esto quiere decir que, desde la estructura misma de la Constitución, se prevé la ocurrencia de conflictos y tensiones tanto entre valores y principios como entre derechos y bienes jurídicos; característica que exige en buena medida que la Corte Constitucional cumpla una función especialmente importante en cuanto a la interpretación y salvaguarda del texto constitucional.

Con el paso de los años y el creciente proceso de apertura económica, uno de esos escenarios de tensión es el que tiene que ver con la celebración de tratados internacionales de inversión que tienen el potencial de afectar de manera directa y grave los derechos e intereses de los pueblos indígenas, quienes son sujetos de especial protección constitucional. Este tipo de tensiones específicas se han podido verificar ya en algunos casos que han llegado a foros internacionales de solución de controversias relativas a la inversión extranjera en Colombia; lo que es una clara evidencia de que se trata de una cuestión a la que debe prestar atención el juez constitucional como intérprete constitucional y como operador jurídico internacional (Lozada Gómez, 2020).

En ese sentido, el control de constitucionalidad de este tipo de tratados se vuelve un escenario particularmente importante para evitar el surgimiento de este tipo de tensiones en la práctica y, con ello, evitar problemas tanto de índole constitucional como económico para el país. Es por ello que las decisiones que 
toma la Corte en estos procesos deben ser analizadas cuidadosamente y, como hemos tratado de hacer brevemente en este documento, a partir de una lectura crítica en clave indígena.

Es así como, a partir del análisis de la jurisprudencia constitucional sobre tratados de inversión en el año 2019 hemos detectado algunos patrones y tendencias que vale la pena tener presentes para procurar un cambio en el razonamiento del juez constitucional. En tal sentido, hemos de destacar que tanto en la sentencia C-252 del 2019 como en la C-254 del 2019 se evidencia claramente que cuando se trata de tratados de contenido económico, el juez constitucional asume una posición más laxa en lo que respecta al análisis constitucional y en particular, en lo que respecta a la participación de los pueblos indígenas en estas decisiones. De esta manera, el juez constitucional no solo defiende la omisión de la consulta previa, sino que además profundiza la exclusión de los pueblos indígenas al omitir su participación activa en los procesos de constitucionalidad y reproduce patrones colonialistas en cuanto al uso del lenguaje y la asimilación de los pueblos indígenas como rúbrica para la exclusión.

\section{Bibliografía}

Acosta Alvarado, P. A. (2018). La naturaleza jurídica de las leyes aprobatorias de tratados y su impacto en el sistema normativo de la Constitución colombiana de 1991. En F. Padrón Pardo y M. Correa Henao. ¿El Estado constitucional en jaque? Tomo 2. El Estado constitucional y el derecho internacional (pp. 165-181). Bogotá: Universidad Externado de Colombia.

Merino, R. (2018). Descolonizar el Derecho, transformar el Estado: fundamentos políticos y legales de la plurinacionalidad. En R. Merino, A. Valencia (eds.). Descolonicar el Derecho. Pueblos indígeneas, derechos humanos y Estado plurinacional (pp. 463-491). Lima: Palestra.

Prieto-Ríos, E. y Rivas-Ramírez, D. (2020). Neocolonialism and the Tension Between International Investment Law and Indignenous Peoples: the Latin American Experience. En J. Borrows y R. Schwartz. Indigenous Peoples and International Trade: Building equitable and inclusive international trade and investment agreements (pp. 85-108). Cambridge: Cambridge University Press. Disponible en: https://doi.org/10.1017/9781108675321.006.

Rivas Ramírez, D. (2017). El derecho internacional de las inversiones, otro de los desaires de la jurisprudencia constitucional colombiana. En J. I. Acosta López, P. A. Acosta Alvarado y D. Rivas Ramírez. De anacronismos y vaticinios: diagnóstico sobre las relaciones entre el derecho internacional y el derecho interno en Latinoamérica (pp. 627-660). Bogotá: Universidad de La Sabana.

- (2018). Un cambio necesario en el control de constitucionalidad de las leyes aprobatorias de tratados internacionales de contenido económico. En F. Padrón 
Pardo y M. Correa Henao. ¿El Estado constitucional en jaque? Tomo 2. El Estado constitucional y el derecho internacional (pp. 217-256). Bogotá: Universidad Externado de Colombia.

- (2020). La recepción del derecho internacional de las inversiones en la Corte Constitucional: breve análisis a partir de su jurisprudencia. En E. H. Fuentes-Contreras y D. Rivas-Ramírez. Método(s) y Derecho(s): Construcciones alternativas de lineas jurisprudenciales para Derechos y garantias. Bogotá: Tirant lo Blanch; Universidad de Bogotá Jorge Tadeo Lozano.

Robledo Silva, P. y Rivas-Ramírez, D. (2019). Cuando la leyenda de El Dorado se hace realidad: Análisis sobre las tensiones entre los intereses económicos y los derechos de los pueblos indígenas. En J. Tole Martínez. Derechos humanos y actividad empresarial en Colombia: implicaciones para el Estado social de Derecho (pp. 21-46). Bogotá: Universidad Externado de Colombia.

- (2020). Pueblos indígenas y derecho internacional de las inversiones: alternativas para una mayor participación. En M. P. García Pachón. Información, participación y justicia ambiental. Herramientas para alcanzar el desarrollo sostenible y la democracia ambiental. Bogotá: Universidad Externado de Colombia.

Tamayo-Álvarez, R. (2020). Constitutionality of the Colombia-France Bilateral Investment Treaty. American Journal of International Law, 114:3, 471-479, https://doi.org/10.1017/ajil.2020.37.

Tole Martínez, J. (2018). La «neutralidad» de la Constitución Económica colombiana. ¿Cómo racionalizar la economía social de mercado con la proliferación de tratados de libre comercio y tratados bilaterales de inversión? En F. Padrón Pardo y M. Correa Henao. ¿El Estado constitucional en jaque? Tomo 2. El Estado constitucional y el derecho internacional (pp. 183-216). Bogotá: Universidad Externado de Colombia.

\section{JURISPRUDENCIA DE LA CORTE CONSTITUCIONAL COLOMBIANA}

Colombia. Corte Constitucional (Sala Plena). Sentencia C-178 de 1995, del 25 de abril de 1995 .

Colombia. Corte Constitucional (Sala Plena). Sentencia C-155 del 2007, del 7 de marzo del 2007.

Colombia. Corte Constitucional (Sala Plena). Sentencia C-309 del 2007, del 3 de mayo del 2007.

Colombia. Corte Constitucional (Sala Plena). Sentencia C-750 del 2008, del 24 de julio del 2008.

Colombia. Corte Constitucional (Sala Cuarta de Revisión). Sentencia T-384A de 2014, del 17 de junio del 2014.

Colombia. Corte Constitucional (Sala Plena). Sentencia C-252 de 2019, del 6 de junio del 2019.

Colombia. Corte Constitucional (Sala Plena). Sentencia C-254 del 2019, del 6 de junio del 2019. 